\title{
Major concerns with the integrity of the mitochondrial ADP/ATP carrier in dodecyl- phosphocholine used for solution NMR studies
}

Martin S. King ${ }^{1}$, Paul G. Crichton ${ }^{1,2}$, Jonathan J. Ruprecht ${ }^{1}$, and Edmund R.S. Kunji ${ }^{1 *}$

${ }^{1}$ Medical Research Council Mitochondrial Biology Unit, University of Cambridge, Cambridge Biomedical Campus, Wellcome Trust / MRC Building, Hills Road, CB2 OXY, Cambridge, United Kingdom

${ }^{2}$ Biomedical Research Centre, Norwich Medical School, University of East Anglia, Norwich Research Park, Norwich NR4 7TJ, United Kingdom.

* Corresponding author; ek@mrc-mbu.cam.ac.uk

Hereby, we wish to note our objections to a paper called "Substrate-modulated ADP/ATP-transporter dynamics revealed by NMR relaxation dispersion" by Brüschweiler et al., published in NSMB in $2015^{1}$. The subject is the yeast mitochondrial ADP/ATP carrier AAC3, which we have studied in great detail ourselves. In particular, we have solved its structure by electron ${ }^{2}$ and $x$-ray crystallography ${ }^{3}$ and have studied its interactions with the specific inhibitors atractyloside (ATR) and carboxyatractyloside (CATR) by single-molecule force spectroscopy ${ }^{4}$. In this paper, the authors claim that AAC 3 can be refolded to homogeneity from inclusion bodies produced in Escherichia coli by using the detergent dodecylphosphocholine (DPC), better known as Foscholine-12 (Anatrace), and that AAC3 is maintained in a folded and active state for the duration of isothermal titration calorimetry (ITC) and NMR experiments. However, in our hands the presence of DPC leads to immediate loss of tertiary structure and inactivation of AAC3 when isolated from the inner membrane of mitochondria, where it was folded and active as shown by functional complementation ${ }^{2,3}$.

To validate the integrity of their refolded protein, the authors first used ITC to measure a Kd of $\sim 15 \mu \mathrm{M}$ for the binding of CATR to DPC-refolded AAC3. They then determined CATR-dependent chemical shift perturbations by NMR, giving a $\mathrm{Kd}$ of $\sim 150 \mu \mathrm{M}$. No other functional assays were done to verify the activity of the refolded protein, such as transport assays. The inhibitors CATR and ATR differ by one carboxylate group and their affinities have been described in 24 different binding studies (Supplementary Table 1). Yet, the authors only compare their values to a Kd of $192 \mu \mathrm{M}$ for the binding of ATR taken from one particular study by Babot et al., $2012^{6}$. Importantly, the $\mu \mathrm{M}$ unit used in this reference was a typographical error and the $\mathrm{Kd}$ value has recently been corrected to $192 \mathrm{nM}$ in an erratum ${ }^{7}$. In fact, published Kd values for CATR binding to AAC in the membrane are all in the low nanomolar range (Supplementary Table 1), consistent with our own measurements that show that ADP transport by AAC3 is half-maximally inhibited by $1.2 \mathrm{nM}$ CATR (Fig. 1a). Thus, the consensus is at least three to four orders of magnitude lower than the Kd values reported for refolded AAC3 in DPC ${ }^{1}$. Our 
bioRxiv preprint doi: https://doi.org/10.1101/329490; this version posted May 25, 2018. The copyright holder for this preprint (which was not certified by peer review) is the author/funder, who has granted bioRxiv a license to display the preprint in perpetuity. It is made available under aCC-BY 4.0 International license.

ITC measurements using native AAC3 from yeast mitochondria purified in dodecylmaltoside/tetraoleoyl cardiolipin gave an average $\mathrm{Kd}$ of $72 \mathrm{nM}$ (Fig. 1b), which is 200 to 2000 -fold lower than those reported in Brüschweiler et al.. These experiments were very difficult to do (only 2 out of 10 trials succeeded), as the enthalpic change is low and the apo-state is very unstable in detergent ${ }^{5}$. There are very few polar side chain interactions in AAC3 that stabilize the structure and they are mainly found on the matrix side, where they form intra-domain rather than inter-domain interactions ${ }^{3}$. The ring of transmembrane $\alpha$-helices is held together largely by the lateral pressure of the membrane and counter pressure from the water-filled cavity (Supplementary Fig. 1a) ${ }^{3}$, explaining why unliganded AAC3 in detergent micelles is prone to unfolding. Binding of CATR introduces a large number of polar and van der Waals interactions, which cross-link most of the transmembrane $\alpha$-helices of AAC3 together ${ }^{3,17}$, explaining the high affinity of CATR for AAC3 as well as the improved stability in detergents (Supplementary Fig. 1b). The experiments reported here and elsewhere clearly show that the Kd of CATR binding to the folded mitochondrial ADP/ATP carrier is in the low nanomolar range, consistent with its role as a powerful toxin. We further note that to obtain the crystal structure, AAC3 was first inhibited with CATR in the mitochondrial membrane, but then solubilized, purified and crystallized in maltoside detergents in the absence of CATR for 5-7 days ${ }^{3}$. Yet, a clear interpretable density for CATR could be observed ${ }^{3}$, demonstrating that the inhibitor remained bound, consistent with extremely low rates of dissociation. The extremely low affinity of CATR to refolded AAC3 in DPC begs the question whether the binding is specific at all. Only a limited number of chemical shift perturbations for CATR binding to refolded AAC3 are observed ${ }^{1}$ and all of them are dynamic, which is inconsistent with tight binding. Moreover, with few exceptions these residues are not near the known CATR binding site nor are they on structural elements that are involved in CATR binding.

We also note that the Kd of ADP binding determined by NMR $(500 \mu \mathrm{M})^{1}$ is $\sim 85$-fold higher than the published consensus values of the carrier in the mitochondrial membrane and $\sim 25$-fold higher than for the solubilized carrier (Supplementary Table 2). Residues that were assigned to have chemical shift perturbations induced by ADP are largely on the matrix side of the carrier, far away from the consensus binding site in the central cavity ${ }^{8-13}$. AAC3 has an isoelectric point of 9.82 , meaning that it is highly positively charged, whereas CATR and the substrates ADP and ATP are negatively charged molecules at neutral $\mathrm{pH}$. The chemical shift perturbations could represent non-specific interactions of CATR or ADP with AAC3 promoted by the high concentrations and temperatures used in these NMR experiments. We are also concerned about the validity of the dynamic studies measured by NMR relaxation dispersion, as CATR binding should lock the protein in a non-dynamic aborted state, which is why would we could solve its structure by crystallography ${ }^{3}$. We also note that the observed dynamics in the presence of substrate do not provide a plausible structural mechanism for transport. 
We have previously demonstrated that DPC is harsh enough to solubilise unfolded mitochondrial carrier protein from E. coli inclusion bodies and is able to denature functional well-folded carrier protein prepared in mild non-ionic detergents ${ }^{14}$. In thermostability assays ${ }^{15}$, AAC3 purified from yeast mitochondrial membranes displayed a typical protein melt curve when diluted into the mild detergent dodecyl-maltoside, consistent with thermal denaturation of a folded protein (Fig. 1c). When CATR was added at a molar ratio of one or above, a marked shift in stability of AAC3 to higher temperatures is observed, consistent with earlier observations ${ }^{14,16}$. When the same AAC3 preparation was diluted into $3 \mathrm{mM} \mathrm{DPC}$, a high signal was observed with no transition, showing that AAC3 in DPC is in a non-native state (Fig. 1d). In this case, CATR addition had no effect, demonstrating that there was no functional binding site. Consistent with these findings, dilution of AAC3 into DPC before reconstitution resulted in a complete loss of CATR-sensitive ADP uptake in liposomes, in contrast to control tests where the protein was diluted into dodecyl-maltoside prior to reconstitution (Supplementary Fig. 2). These observations clearly demonstrate that AAC3 is soluble but in a non-native state in DPC. In conclusion, we believe that the data presented by Brüschweiler et al. have no biological relevance.

\section{Acknowledgements}

We would like to acknowledge support by the intramural programme MC_UU_00015/1 of the Medical Research Council, UK.

\section{References}

1. Bruschweiler, S., Yang, Q., Run, C. \& Chou, J.J. Substrate-modulated ADP/ATP-transporter dynamics revealed by NMR relaxation dispersion. Nat Struct Mol Biol 22, 636-41 (2015).

2. Kunji, E.R.S. \& Harding, M. Projection structure of the atractyloside-inhibited mitochondrial ADP/ATP carrier of Saccharomyces cerevisiae. J. Biol. Chem. 278, 36985-36988 (2003).

3. Ruprecht, J.J. et al. Structures of yeast mitochondrial ADP/ATP carriers support a domain-based alternating-access transport mechanism. Proc. Natl. Acad. Sci. U.S.A 111, E426-E434 (2014).

4. Kedrov, A. et al. Probing the interactions of carboxy-atractyloside and atractyloside with the yeast mitochondrial ADP/ATP Carrier. Structure 18, 39-46 (2010).

5. Babot, M., Blancard, C., Zeman, I., Lauquin, G.J. \& Trezeguet, V. Mitochondrial ADP/ATP carrier: preventing conformational changes by point mutations inactivates nucleotide transport activity. Biochemistry 51, 7348-56 (2012). 
6. Babot, M., Blancard, C., Zeman, I., Lauquin, G.J. \& Trezeguet, V. Correction to Mitochondrial ADP/ATP Carrier: Preventing Conformational Changes by Point Mutations Inactivates Nucleotide Transport Activity. Biochemistry 55, 2422 (2016).

7. Bamber, L., Harding, M., Butler, P.J.G. \& Kunji, E.R.S. Yeast mitochondrial ADP/ATP carriers are monomeric in detergents. Proc. Natl. Acad. Sci. U.S.A. 103, 16224-16229 (2006).

8. Kunji, E.R.S. \& Robinson, A.J. The conserved substrate binding site of mitochondrial carriers. Biochimica Biophysica Acta 1757, 1237-48 (2006).

9. Robinson, A.J. \& Kunji, E.R.S. Mitochondrial carriers in the cytoplasmic state have a common substrate binding site. Proc. Natl. Acad. Sci. U.S.A. 103, 2617-2622 (2006).

10. Robinson, A.J., Overy, C. \& Kunji, E.R.S. The mechanism of transport by mitochondrial carriers based on analysis of symmetry. Proc. Natl. Acad. Sci. U.S.A. 105, 17766-17771 (2008).

11. Dehez, F., Pebay-Peyroula, E. \& Chipot, C. Binding of ADP in the mitochondrial ADP/ATP carrier is driven by an electrostatic funnel. J. Am. Chem. Soc. 130, 12725-12733 (2008).

12. Mifsud, J. et al. The substrate specificity of the human ADP/ATP carrier AAC1. Mol. Membr. Biol. 30, $160-168$ (2013).

13. Wang, Y. \& Tajkhorshid, E. Electrostatic funneling of substrate in mitochondrial inner membrane carriers. Proc. Natl. Acad. Sci. U.S.A. 105, 9598-9603 (2008).

14. Crichton, P.G. et al. Trends in thermostability provide information on the nature of substrate, inhibitor, and lipid interactions with mitochondrial carriers. J. Biol. Chem. 290, 8206-8217 (2015).

15. Alexandrov, A.I., Mileni, M., Chien, E.Y., Hanson, M.A. \& Stevens, R.C. Microscale fluorescent thermal stability assay for membrane proteins. Structure 16, 351-9 (2008).

16. King, M.S., Kerr, M., Crichton, P.G., Springett, R. \& Kunji, E.R. Formation of a cytoplasmic salt bridge network in the matrix state is a fundamental step in the transport mechanism of the mitochondrial ADP/ATP carrier. Biochim. Biophys. Acta 1857, 14-22 (2016).

17. Pebay-Peyroula, E. et al. Structure of mitochondrial ADP/ATP carrier in complex with carboxyatractyloside. Nature 426, 39-44 (2003). 

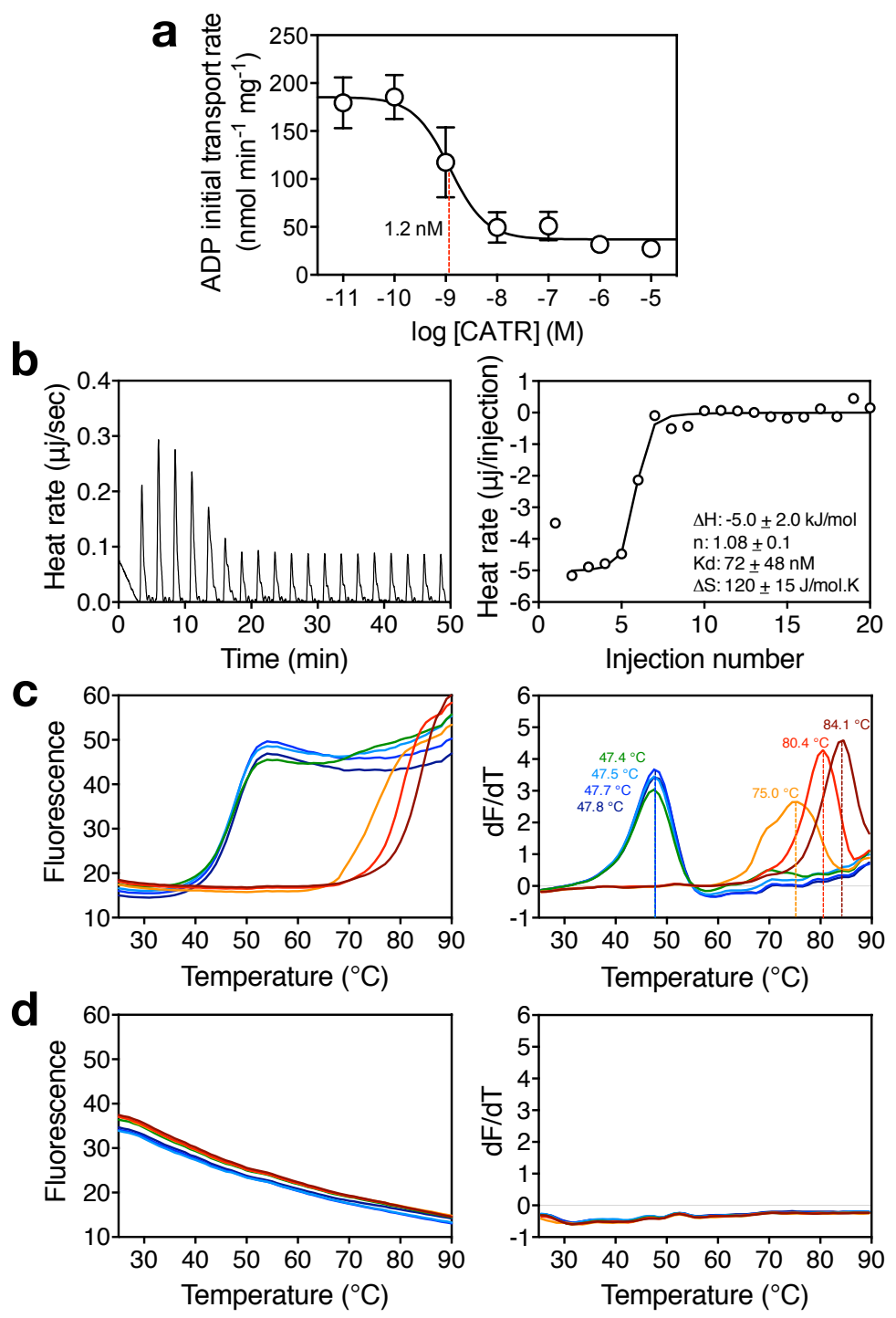

Figure 1. CATR binding and stability of the yeast mitochondrial ADP/ATP carrier AAC3 in dodecylmaltoside and dodecyl-phosphocholine. (a) Inhibition of ADP transport by the yeast mitochondrial ADP/ATP carrier AAC3 by carboxyatractyloside. AAC3 purified from yeast mitochondria in dodecylmaltoside was reconstituted into liposomes. The CATR concentration required for half-maximal inhibition was determined by measuring the initial ADP uptake rate at different concentrations of CATR $(n=3)$. (b) Isothermal titration calorimetry: enthalpy changes associated with the titration of CATR into $A A C 3$ at $10{ }^{\circ} \mathrm{C}$ (left panel), and corresponding isotherms fitted to a one site binding model with $\Delta \mathrm{H}, \mathrm{Kd}$, stoichiometry and $\Delta S$ as fitting parameters (right panel). (Inset) Average data ( $\pm S D$ ) from two titration

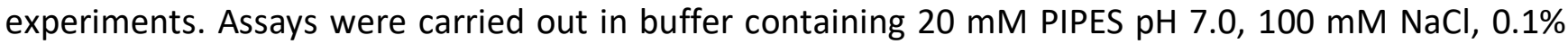
dodecyl-maltoside, $0.1 \mathrm{mg} \mathrm{mL}^{-1}$ tetraoleoyl cardiolipin. (c) Thermostability of the yeast mitochondrial ADP/ATP carrier AAC3 diluted into dodecyl-maltoside in the presence of different amounts of CATR (left panel) and derivatives (right panel). (d) same as (c) but with AAC3 diluted into dodecyl-phosphocholine. The temperature of the protein sample is increased from 25 to $90{ }^{\circ} \mathrm{C}$ while protein unfolding is monitored with the fluorophore $\mathrm{N}$-[4-(7-diethylamino-4-methyl-3-coumarinyl)phenyl] maleimide $(C P M)^{15}$. CPM reacts with protein thiols as they become solvent-exposed due to denaturation of the protein to give a fluorescent adduct. The CATR:AAC3 molar ratios were 0 (black), 0.001 (dark blue), 0.01 (light blue), 0.1 (green), 1 (orange), and 10 (red), and 100 (dark red). The protein concentration was approximately $1 \mu \mathrm{M}$ AAC3. 


\section{Supplementary Note}

\section{Major concerns with the integrity of the mitochondrial ADP/ATP carrier in dodecyl- phosphocholine used for solution NMR studies}

Martin S. King ${ }^{1}$, Paul G. Crichton ${ }^{1,2}$, Jonathan J. Ruprecht ${ }^{1}$, and Edmund R.S. Kunji ${ }^{{ }^{*}}$

${ }^{1}$ Medical Research Council, Mitochondrial Biology Unit, Cambridge Biomedical Campus,

Wellcome Trust / MRC Building, Hills Road, CB2 OXY, Cambridge, United Kingdom

${ }^{2}$ Biomedical Research Centre, Norwich Medical School, University of East Anglia, Norwich Research Park, Norwich NR4 7TJ, United Kingdom.

* Corresponding author; ek@mrc-mbu.cam.ac.uk

\section{Supplementary Materials and Methods}

\section{Construction of yeast AAC3 expression strains}

The gene coding for AAC3 of Saccharomyces cerevisiae was cloned into the yeast expression vector pYES-PMIR2-AAC2 with an N-terminal eight-histidine tag and Factor Xa cleavage site ${ }^{18}$. Expression vectors were transformed by electroporation into S. cerevisiae strain WB12 (MAT $\alpha$ ade2-1 trp1-1 ura31 can1-100 aac1::LEU2 aac2::HIS3) ${ }^{19}$, which lacks functional Aac1p and Aac2 $p$ carriers. Transformants were selected initially on SC medium minus tryptophan plates, and then on YPG plates, confirming they expressed functional ADP/ATP carriers through complementation.

\section{Preparation of lipid for protein purification}

Tetraoleoyl cardiolipin (18:1) dissolved in chloroform was purchased from Avanti Polar Lipids (Alabaster, Alabama). Typically, $100 \mathrm{mg}$ of lipid was dispensed into a glass vial, and chloroform was removed by evaporation under a stream of nitrogen gas. Lipids were solubilized in $10 \%(\mathrm{w} / \mathrm{v})$ dodecylmaltoside by vortexing for $4 \mathrm{~h}$ at room temperature to give a $10 \mathrm{mg} \mathrm{mL}^{-1}$ lipid in $10 \%$ detergent stock. The stocks were snap-frozen and stored in liquid nitrogen.

\section{Purification of AAC3}

A 5-L pre-culture was used to inoculate $50 \mathrm{~L}$ of YPG medium in an Applikon 140 Pilot System with an eZ controller. Cells were grown at $30{ }^{\circ} \mathrm{C}$ for $72 \mathrm{~h}$, and harvested by centrifugation $\left(4,000 \mathrm{~g}, 20 \mathrm{~min}, 4{ }^{\circ} \mathrm{C}\right)$. Mitochondria were prepared with established methods ${ }^{18}$, flash frozen in liquid nitrogen, and stored at $-80{ }^{\circ} \mathrm{C}$ until use. Yeast mitochondria (1 g total protein) were solubilized in $2 \%$ dodecyl-maltoside (Glycon) dissolved in a buffer consisting of $20 \mathrm{mM}$ imidazole, $150 \mathrm{mM} \mathrm{NaCl}, 20 \mathrm{mM}$ PIPES, pH 7.0, and 
an EDTA-free complete protease inhibitor tablet (Roche Diagnostics Ltd) by mixing at $4{ }^{\circ} \mathrm{C}$ for one hour. Particulate material was removed by ultracentrifugation $\left(140,000 \mathrm{~g}, 45 \mathrm{~min}, 4^{\circ} \mathrm{C}\right)$. The soluble fraction was loaded onto a Ni-Sepharose high performance column (Amersham Biosciences) at $1 \mathrm{~mL} \mathrm{~min}^{-1}$ on an ÄKTAprime (GE Healthcare). The column was washed with 40 column volumes of buffer containing $20 \mathrm{mM}$ PIPES pH 7.0, $150 \mathrm{mM} \mathrm{NaCl}, 20 \mathrm{mM}$ imidazole, $0.1 \%$ dodecyl-maltoside, $0.1 \mathrm{mg} \mathrm{mL}^{-1}$ tetraoleoyl cardiolipin. The column material was washed with a further 20 column volumes of buffer B containing $20 \mathrm{mM}$ PIPES pH 7.0, $100 \mathrm{mM} \mathrm{NaCl}, 0.1 \%$ dodecyl-maltoside, $0.1 \mathrm{mg} \mathrm{mL}^{-1}$ tetraoleoyl cardiolipin. The column material was resuspended with $500 \mu \mathrm{L}$ buffer $\mathrm{B}$ and transferred to a vial containing $5 \mathrm{mM} \mathrm{CaCl}_{2}$ and $75 \mu \mathrm{L}$ Factor Xa (New England BioLabs), vortexed thoroughly, and incubated at $10^{\circ} \mathrm{C}$ overnight. The following day, the cleaved protein was separated from the nickel sepharose media using filtration and centrifugation, and the protein concentration determined and the protein snap-frozen and stored in liquid nitrogen.

\section{Thermostability analysis}

Thermostability data were obtained by using the thiol-reactive fluorophore N-[4-(7-diethylamino-4methyl-3-coumarinyl)phenyl] maleimide (CPM), which undergoes an increase in fluorescence emission following reaction with cysteine residues ${ }^{20}$. A modified procedure using a rotary qPCR machine was used, as described previously ${ }^{21}$. For this purpose, a $5 \mathrm{mg} \mathrm{mL}^{-1}$ stock of CPM dissolved in DMSO was diluted 50-fold into buffer containing $20 \mathrm{mM}$ PIPES pH 7.0, $100 \mathrm{mM} \mathrm{NaCl}, 0.1 \%$ dodecyl-maltoside and $0.1 \mathrm{mg} \mathrm{mL}^{-1}$ tetraoleoyl cardiolipin, vortexed and the solution was allowed to equilibrate in the dark at room temperature for $10 \mathrm{~min}$. Approximately $1.5 \mu \mathrm{g}$ of purified protein was added into a final volume of $45 \mu \mathrm{L}$ buffer containing either $20 \mathrm{mM}$ PIPES pH 7.0, $100 \mathrm{mM} \mathrm{NaCl}, 0.1 \%$ dodecyl-maltoside, $0.1 \mathrm{mg}$ $\mathrm{mL}^{-1}$ tetraoleoyl cardiolipin (for the dodecyl-maltoside assays) or $20 \mathrm{mM}$ PIPES pH 7.0, $100 \mathrm{mM} \mathrm{NaCl}, 3$ $\mathrm{mM}$ dodecyl-phosphocholine (for the dodecyl-phosphocholine assays), and $5 \mu \mathrm{L}$ CPM working solution was added, and the solution was vortexed and allowed to equilibrate in the dark for 10 min at room temperature in the presence of increasing concentrations of carboxyatractyloside. Fluorescence of the CPM dye was measured on a Qiagen Rotorgene $Q$ using the HRM channel, which provides excitation light at 440-480 nm with emission detected at 505-515 nm. Measurements were made in $1{ }^{\circ} \mathrm{C}$ intervals from $25-90^{\circ} \mathrm{C}$ with a 'wait between reading' set to $4 \mathrm{~s}$, which equated to a ramp rate of $5.6^{\circ} \mathrm{C} / \mathrm{min}$, following an initial pre-incubation step of 90 seconds. Data were analyzed and melting temperatures, the peak in the derivative of the melting curve, were determined with the software supplied with the instrument. 
The enthalpy changes associated with carboxyatractyloside binding to purified AAC3 were recorded with a NanoITC-LV isothermal titration calorimeter (TA Instruments) at $10^{\circ} \mathrm{C}$. Both carboxyatractyloside titrant and protein samples were degassed under vacuum at $10^{\circ} \mathrm{C}$ for at least 20 min before the titration experiment. Carboxyatractyloside (500 $\mu \mathrm{M}$ stock prepared in a buffer containing $20 \mathrm{mM}$ PIPES pH 7.0, $100 \mathrm{mM} \mathrm{NaCl}, 0.1 \%$ dodecyl-maltoside and $0.1 \mathrm{mg} \mathrm{mL}^{-1}$ tetraoleoyl cardiolipin) was titrated into purified $25 \mu \mathrm{M}$ AAC3 (0.83 $\mathrm{mg} \mathrm{mL}^{-1}$ in the same buffer) in $2-\mu \mathrm{L}$ injections in an initial volume of $170 \mu \mathrm{L}$ at 3-min intervals with a stirrer speed of $350 \mathrm{rpm}$. Isotherms were analysed by using the instrument software (NanoAnalyze) and fitted to a one-site binding model with $\Delta \mathrm{H}, \mathrm{Kd}$, and stoichiometry as fitting parameters.

\section{Reconstitution}

L- $\alpha$-phosphatidylcholine and tetraoleoyl cardiolipin were mixed in 20:1 (w/w) ratio (10 $\mathrm{mg}$ total lipid) in chloroform and dried under a nitrogen stream, dissolved in methanol, and re-dried to a smear in a 1.5-mL tube. The lipid was emulsified in a buffer solution and solubilised with $55 \mu \mathrm{L} 25 \%(\mathrm{v} / \mathrm{v})$ pentaethylene glycol monodecyl detergent (Sigma) on ice before addition of $30 \mu \mathrm{g}$ of purified AAC3

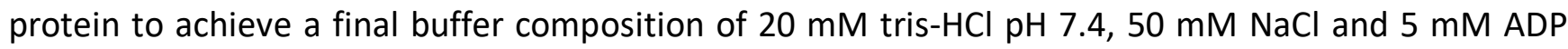
('internal buffer'), with a volume equivalent to $0.6 \mathrm{~mL}$ in the absence of detergent. The purified protein was either added directly to the lipid/detergent reconstitution mix or, for testing the influence of detergents on protein integrity, first diluted 20 -fold into $0.1 \%$ dodecyl-maltoside or $0.1 \%$ dodecylphosphocholine and incubated on ice (30 mins) before addition. To form the proteoliposomes, the detergent was stepwise removed from the reconstitution mix through addition of $4 \times 30 \mathrm{mg}$ and $4 \times 60$ mg of Bio-Beads SM-2 in 20-min intervals with gentle mixing in a cold room overnight. The resulting proteoliposome suspension was separated from the biobeads using an empty spin column (Bio-Rad, Hemel Hempstead, UK) and exchanged into 'external buffer' (20 mM tris- $\mathrm{HCl}$ pH 7.4, $50 \mathrm{mM} \mathrm{NaCl}$, without ADP) using a PD10 desalting column (GE Healthcare, Little Chalfont, UK). The proteoliposomes were eluted in $1.5 \mathrm{~mL}$ and diluted a further 9-fold in external buffer before ADP uptake assays.

\section{ADP uptake assays}

Uptake assays were carried out using a Hamilton MicroLab Star robot (Hamilton Robotics Ltd., Birmingham, UK) with $100 \mu \mathrm{L}$ of the proteoliposome suspension added to each well of a MultiScreenHTS-HA 96-well filter plate (pore size $=0.45 \mu \mathrm{m}$ Millipore, Billerica, USA), with CATR inhibitor added where required. Uptake of radiolabeled ADP was initiated by the addition of $100 \mu \mathrm{L}$ of external buffer containing $\left[{ }^{14} \mathrm{C}\right]-\mathrm{ADP}\left(2.2 \mathrm{GBq} \mathrm{mmol}^{-1}\right)$ to give a final working concentration of $1.5 \mu \mathrm{M}$ $\left[{ }^{14} \mathrm{C}\right]-\mathrm{ADP}$ in the assay. The transport was stopped at $0,10,20,30,45 \mathrm{~s}, 1,2.5,5,7.5,10$ and 15 min by 
the addition of $200 \mu \mathrm{L}$ ice-cold $20 \mathrm{mM}$ tris- $\mathrm{HCl}$ buffer and rapid filtration using a vacuum manifold, followed by an additional wash step with $200 \mu \mathrm{L}$ ice-cold external buffer and repeated filtration. The amount of $\left[{ }^{14} \mathrm{C}\right]-\mathrm{ADP}$ accumulated in the liposomes was measured by the addition of $200 \mu \mathrm{L}$ MicroScint20 (Perkin Elmer, Waltham, USA) and quantifying the amount of radioactivity with the TopCount scintillation counter (Perkin Elmer, Waltham, USA). Specific initial uptake rates were calculated using the amount of protein reconstituted into liposomes.

Curves for each uptake were fitted to a 'plateau and one-phase association' relationship in Prism (GraphPad). Initial rates were determined from the fit of the entire curve over the $15 \mathrm{~min}$. There is a delay between the addition of radioactive substrate and the washing/vacuuming steps for the zero time point due to the filtration time. For this reason, the fit was not restrained to go through the origin, and the delay was estimated to be $15 \mathrm{~s}$.

\section{Supplementary figures}
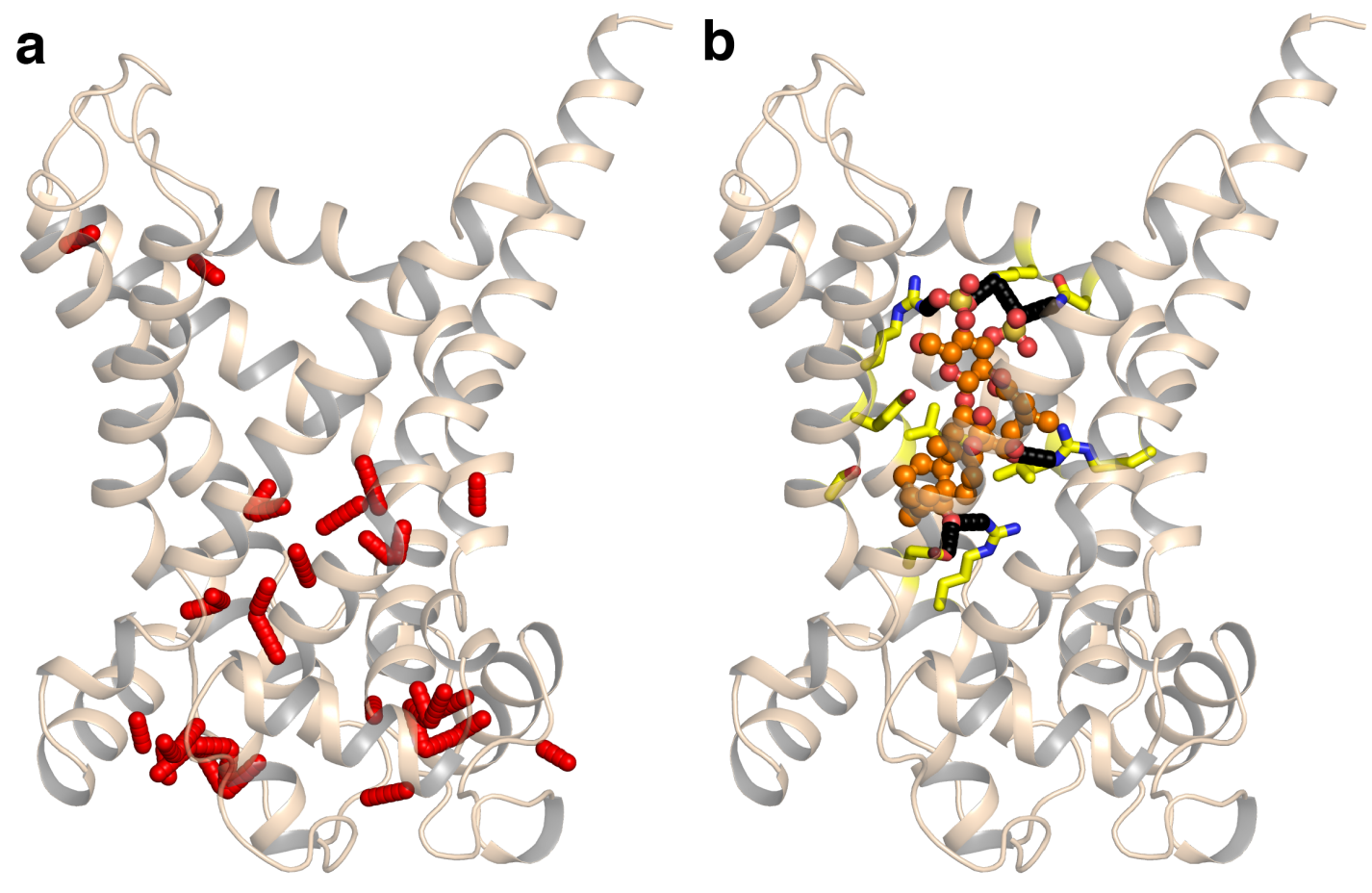

Supplementary Figure 1. Structural stability of AAC3. (a) The inter-side chain interactions (red sticks) in the structure of AAC3 (cartoon) ${ }^{22}$. There are far fewer polar side-chain interactions between the transmembrane $\alpha$-helices than on the matrix side, and most of them are between residues of the matrix salt bridge network ${ }^{22,23}$ (b) Polar interactions (black sticks) of carboxyatractyloside (orange ball and stick) with AAC3 (cartoon). 

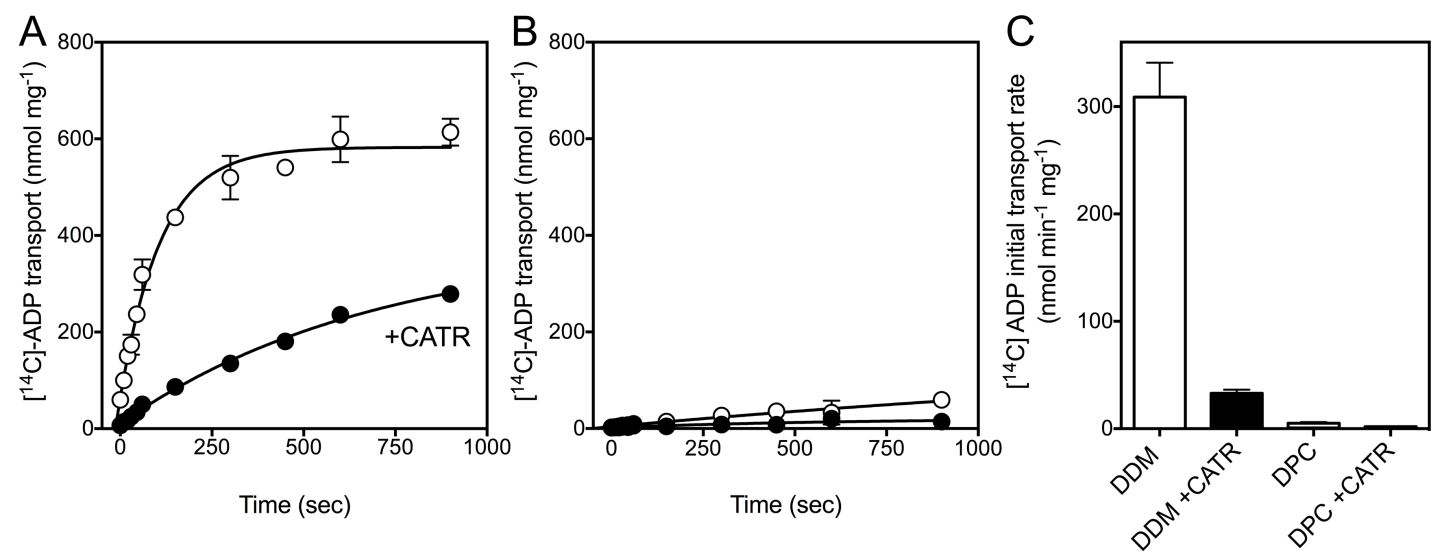

Supplementary Figure 2. Dodecyl-phosphocholine inactivates the yeast mitochondrial ADP/ATP carrier AAC3. ${ }^{14}$ C-ADP uptake by AAC3 reconstituted into liposomes from (a) dodecyl-maltoside (DDM) or (b) dodecylphosphocholine (DPC). (c) Average initial uptakes rates of experiments in (a) and (b) (n=3). CATR is carboxy-atractyloside. 


\section{Supplementary Tables}

Supplementary Table 1. Previously reported values for the dissociation constant (Kd) of carboxyatractyloside (CATR) and atractyloside (ATR) binding to the mitochondrial ADP/ATP carrier

\begin{tabular}{|c|c|c|c|}
\hline Inhibitor & $\mathrm{Kd}(\mathrm{nM})$ & Notes & Reference \\
\hline CATR & 12,15 & bovine $A A C$ in heart mitochondria & 24 \\
\hline CATR & 310 & solubilised bovine AAC in LAPAO & 24 \\
\hline CATR & 10 & reconstituted bovine $A A C$ & 24 \\
\hline CATR & 10 & bovine heart mitochondria & 25 \\
\hline CATR & $5-10$ & rat $A A C$ in liver mitochondria & 26 \\
\hline CATR & 5 & reconstituted bovine AAC & 27 \\
\hline CATR & $<10$ & bovine $A A C$ in heart mitochondria & 28 \\
\hline ATR & 50 & bovine $A A C$ in heart mitochondria & 25 \\
\hline ATR & 375 & yeast Aac $2 p$ in mitochondria & 29 \\
\hline ATR & $150-200$ & yeast Aac $2 p$ in mitochondria & 30 \\
\hline ATR & $100-150$ & yeast Aac2p in mitochondria & 31 \\
\hline ATR & 270 & yeast Aac $2 p$ in mitochondria & 32 \\
\hline ATR & 220 & yeast Aac $2 p$ in mitochondria & 33 \\
\hline ATR & 327 & yeast Aac2p in mitochondria & 34 \\
\hline ATR & 192 & yeast Aac2p in mitochondria & 35 \\
\hline ATR & 165 & bovine AAC in heart mitochondria & 36 \\
\hline ATR & 70 & rat $A A C$ in liver mitochondria & 36 \\
\hline ATR & 11 & rat AAC liver mitochondria & 37 \\
\hline ATR & 30 & bovine $A A C$ in heart mitochondria & 38 \\
\hline ATR & 100 & bovine $A A C$ in heart mitochondria & 38 \\
\hline ATR & 100 & yeast Aac2p in mitochondria & 39 \\
\hline ATR & 34 & human $A A C 1$ in yeast mitochondria & 39 \\
\hline ATR & 22 & human $A A C 2$ in yeast mitochondria & 39 \\
\hline ATR & 49 & human AAC3 in yeast mitochondria & 39 \\
\hline ATR & 192 & yeast Aac $2 p$ in mitochondria & 40,41 \\
\hline
\end{tabular}


Supplementary Table 2: Previously reported values for the dissociation (Kd) constants of ADP and ATP to the mitochondrial ADP/ATP carrier

\begin{tabular}{|c|c|c|c|}
\hline Substrate & $\mathrm{Kd}(\mu \mathrm{M})$ & Notes & Reference \\
\hline ADP & 7 & bovine $A A C$ in heart mitochondria & 24 \\
\hline ADP & 6.6 & bovine $A A C$ in heart mitochondria & 42 \\
\hline ADP & 7 & bovine $A A C$ in heart mitochondria & 43 \\
\hline ADP & 4.1 & rat $A A C$ in heart mitochondria & 43 \\
\hline ADP & 4 & bovine $A A C$ in heart mitochondria & 38 \\
\hline ADP & 2.7 & bovine $A A C$ in heart mitochondria & 44 \\
\hline ADP & 10 & bovine $A A C$ in heart mitochondria & 45 \\
\hline ATP & 12 & bovine AAC in heart mitochondria & 24 \\
\hline ATP & 12 & bovine AAC in heart mitochondria & 43 \\
\hline ATP & 3 & bovine $A A C$ in heart mitochondria & 38 \\
\hline ATP & 5 & bovine $A A C$ in heart mitochondria & 28 \\
\hline ADP & 20 & Solubilised bovine AAC1 & 46 \\
\hline ATP & 20 & Solubilised bovine AAC1 & 46 \\
\hline
\end{tabular}

\section{References}

18. Kunji, E.R.S. \& Harding, M. Projection structure of the atractyloside-inhibited mitochondrial ADP/ATP carrier of Saccharomyces cerevisiae. J. Biol. Chem. 278, 36985-36988 (2003).

19. Hashimoto, M. et al. Expression of the bovine heart mitochondrial ADP/ATP carrier in yeast mitochondria: significantly enhanced expression by replacement of the $\mathrm{N}$-terminal region of the bovine carrier by the corresponding regions of the yeast carriers. Biochim. Biophys. Acta 1409, 113124 (1999).

20. Alexandrov, A.I., Mileni, M., Chien, E.Y., Hanson, M.A. \& Stevens, R.C. Microscale fluorescent thermal stability assay for membrane proteins. Structure 16, 351-359 (2008).

21. Crichton, P.G. et al. Trends in thermostability provide information on the nature of substrate, inhibitor and lipid interactions with mitochondrial carriers. J. Biol. Chem. 290, 8206-8217 (2015).

22. Ruprecht, J.J. et al. Structures of yeast mitochondrial ADP/ATP carriers support a domain-based alternating-access transport mechanism. Proc. Natl. Acad. Sci. U.S.A 111, E426-E434 (2014). 
23. Pebay-Peyroula, E. et al. Structure of mitochondrial ADP/ATP carrier in complex with carboxyatractyloside. Nature 426, 39-44 (2003).

24. Kramer, R. \& Klingenberg, M. Reconstitution of inhibitor binding properties of the isolated ADP/ATP carrier-linked binding protein. Biochemistry 16, 4954-4961 (1977).

25. Klingenberg, M., Grebe, K. \& Scherer, B. The binding of atractylate and carboxyatractylate to mitochondria. Eur. J. Biochem. 52, 351-363 (1975).

26. Vignais, P.V., Vignais, P.M. \& Defaye, G. Adenosine diphosphate translocation in mitochondria. Nature of the receptor site for carboxyatractyloside (gummiferin). Biochemistry 12, 1508-1519 (1973).

27. Kramer, R. Interaction of membrane-surface charges with the reconstituted ADP/ATP carrier from mitochondria. Biochim Biophys Acta 735, 145-159 (1983).

28. Dupont, Y., Brandolin, G. \& Vignais, P.V. Exploration of the nucleotide binding-sites of the isolated ADP/ATP carrier protein from beef-heart mitochondria .1. Probing of the nucleotide sites by naphthoyl-ATP, a fluorescent nontransportable analog of ATP. Biochemistry 21, 6343-6347 (1982).

29. Clemencon, B., Rey, M., Trezeguet, V., Forest, E. \& Pelosi, L. Yeast ADP/ATP carrier isoform 2 J. Biol. Chem. 286, 36119-36131 (2011).

30. Brandolin, G., Le Saux, A., Trezeguet, V., Vignais, P.V. \& Lauquin, G.J. Biochemical characterisation of the isolated Anc2 adenine nucleotide carrier from Saccharomyces cerevisiae mitochondria. Biochem. Biophys. Res. Commun. 192, 143-150 (1993).

31. Fiore, C. et al. Purification of histidine-tagged mitochondrial ADP/ATP carrier: influence of the conformational states of the C-terminal region. Protein Expr. Purif. 19, 57-65 (2000).

32. Dahout-Gonzalez, C., Ramus, C., Dassa, E.P., Dianoux, A.C. \& Brandolin, G. Conformationdependent swinging of the matrix loop $\mathrm{m} 2$ of the mitochondrial Saccharomyces cerevisiae ADP/ATP carrier. Biochemistry 44, 16310-16320 (2005).

33. Zeman, I. et al. Four mutations in transmembrane domains of the mitochondrial ADP/ATP carrier increase resistance to bongkrekic acid. J. Bioenerg. Biomembr. 35, 243-56 (2003).

34. David, C. et al. Two residues of a conserved aromatic ladder of the mitochondrial ADP/ATP carrier are crucial to nucleotide transport. Biochemistry 47, 13223-13231 (2008).

35. Babot, M., Blancard, C., Pelosi, L., Lauquin, G.J. \& Trezeguet, V. The transmembrane prolines of the mitochondrial ADP/ATP carrier are involved in nucleotide binding and transport and its biogenesis. J. Biol. Chem. 287, 10368-10378 (2012).

36. Klingenberg, M., Falkner, G., Erdelt, H. \& Grebe, K. On the relation between adenine nucleotide carrier sites and atractyloside binding in mitochondria. FEBS Lett. 16, 296-300 (1971). 
37. Vignais, P.V., Vignais, P.M. \& Colomb, M.G. S-35 atractyloside binding affinity to inner mitochondrial membrane. FEBS Lett. 8, 328-332 (1970).

38. Scherer, B. \& Klingenberg, M. Demonstration of the relationship between the adenine nucleotide carrier and the structural changes of mitochondria as induced by adenosine 5'-diphosphate. Biochemistry 13, 161-170 (1974).

39. De Marcos Lousa, C., Trezeguet, V., Dianoux, A.C., Brandolin, G. \& Lauquin, G.J. The human mitochondrial ADP/ATP carriers: kinetic properties and biogenesis of wild-type and mutant proteins in the yeast S. cerevisiae. Biochemistry 41, 14412-14420 (2002).

40. Babot, M., Blancard, C., Zeman, I., Lauquin, G.J.M. \& Trezeguet, V. Mitochondrial ADP/ATP carrier: preventing conformational changes by point mutations inactivates nucleotide transport activity. Biochemistry 51, 7348-7356 (2012).

41. Babot, M., Blancard, C., Zeman, I., Lauquin, G.J. \& Trezeguet, V. Correction to Mitochondrial ADP/ATP Carrier: Preventing Conformational Changes by Point Mutations Inactivates Nucleotide Transport Activity. Biochemistry 55, 2422 (2016).

42. Weidemann, M.J., Erdelt, H. \& Klingenberg, M. Effect of bongkrekic acid on the adenine nucleotide carrier in mitochondria: tightening of adenine nucleotide binding and differentiation between inner and outer sites. Biochem. Biophys. Res. Commun. 39, 363-370 (1970).

43. Weidemann, M.J., Erdelt, H. \& Klingenberg, M. Adenine nucleotide translocation of mitochondria. Identification of carrier sites. Eur. J. Biochem. 16, 313-335 (1970).

44. Klingenberg, M. \& Buchholz, M. On the mechanism of bongkrekate effect on the mitochondrial adenine-nucleotide carrier as studied through the binding of ADP. Eur. J. Biochem. 38, 346-358 (1973).

45. Erdelt, H., Weidemann, M.J., Buchholz, M. \& Klingenberg, M. Some principle effects of bongkrekic acid on the binding of adenine nucleotides to mitochondrial membranes. Eur. J. Biochem. 30, 107122 (1972).

46. Klingenberg, M., Mayer, I. \& Appel, M. Interaction of fluorescent 3'-[1,5(dimethylamino)naphthoyl]adenine nucleotides with the solubilized ADP/ATP carrier. Biochemistry 24, 3650-3659 (1985). 